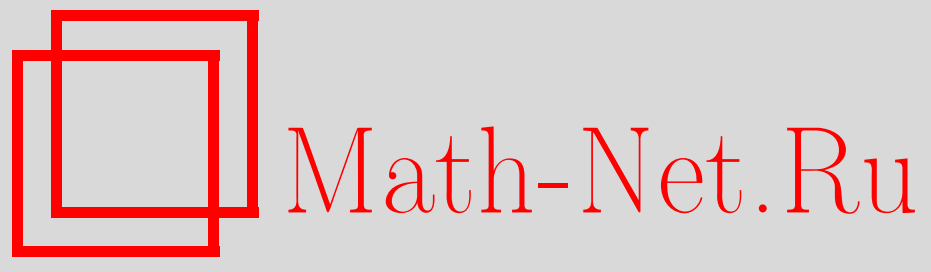

Е. А. Кудрявцева, Об инвариантах сопряженности на группе, сохраняющих площади диффеоморфизмов круга, Матем. заметки, 2014, том 95, выпуск 6, 951-954

DOI: https://doi.org/10.4213/mzm10459

Использование Общероссийского математического портала Math-Net.Ru подразумевает, что вы прочитали и согласны с пользовательским соглашением http://www . mathnet.ru/rus/agreement

Параметры загрузки:

IP : 54.89 .56 .158

26 апреля 2023 г., 10:45:18

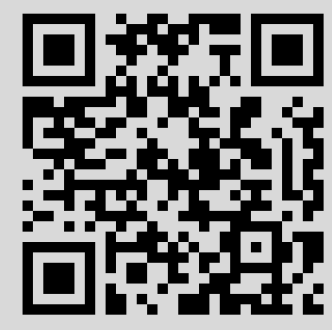




\section{Об инвариантах сопряженности на группе сохраняющих площади диффеоморфизмов круга}

\section{Е. А. Кудрявцева}

Пусть $\mathscr{G}=S \operatorname{Diff}\left(B^{2}\right)$ - группа диффеоморфизмов круга $B^{2}=\left\{(x, y) \in \mathbb{R}^{2} \mid x^{2}+y^{2} \leqslant 1\right\}$ на себя, сохраняющих форму площади $\omega=d x \wedge d y$, т.е. группа симплектоморфизмов круга $\left(B^{2}, \omega\right)$. В настоящей работе изучаются инварианты сопряженности на группе $\widetilde{\mathscr{G}}-$ универсальной накрывающей группы $\mathscr{G}$. Мы доказываем, что любой инвариант сопряженности на $\widetilde{\mathscr{G}}$, имеющий регулярную и $C^{1}$-непрерывную производную, выражается через инвариант Калаби. Перейдем к точным формулировкам.

1. Инварианты сопряженности на группе $\widetilde{\mathscr{G}}$. Рассмотрим группу $\mathscr{G}=S \operatorname{Diff}\left(B^{2}\right)$ сохраняющих площади диффеоморфизмов (т.е. симплектоморфизмов) круга с $C^{\infty}$-топологией. Эта группа линейно связна, так как она является (сильным деформационным) ретрактом [1] линейно связной [2] группы $\operatorname{Diff}\left(B^{2}\right)$ сохраняющих ориентацию диффеоморфизмов. Более того, для любого симплектоморфизма $\varphi \in \mathscr{G}$ существует кусочно-гладкое семейство симплектоморфизмов $\varphi_{t} \in \mathscr{G}, 0 \leqslant t \leqslant 1$, такое, что $\varphi_{0}=\operatorname{id}_{B^{2}}$ и $\varphi_{1}=\varphi$. Ясно, что при любом $t \in[0,1]$ поле скоростей $(d / d t) \varphi_{t}$ является гамильтоновым на $\left(B^{2}, \omega\right)$ с функцией Гамильтона $H_{t} \in C^{\infty}\left(B^{2}\right)$, постоянной на окружности $\partial B^{2}$. Без ограничения общности мы можем и будем считать, что $\left.H_{t}\right|_{\partial B^{2}}=0$; обозначим множество гладких функций на $B^{2}$ с этим свойством через $C_{0}^{\infty}\left(B^{2}, \partial B^{2}\right)$. Итак, рассматриваемое семейство симплектоморфизмов $\varphi_{t} \in \mathscr{G}$ эквивалентным образом задается (кусочно-гладким) семейством функций

$$
H_{t} \in C_{0}^{\infty}\left(B^{2}, \partial B^{2}\right), \quad 0 \leqslant t \leqslant 1 \text {. }
$$

Универсалъную накрывающую $\widetilde{\mathscr{G}}$ группы $\mathscr{G}$ можно определить так. Любой ее элемент $\widetilde{\varphi}$ задается (кусочно-гладким) семейством симплектоморфизмов $\varphi_{t} \in \mathscr{G}, 0 \leqslant t \leqslant 1$, таким, что $\varphi_{0}=\operatorname{id}_{B^{2}}$. Два семейства $\varphi_{t}, \psi_{t} \in \mathscr{G}, 0 \leqslant t \leqslant 1$, с $\varphi_{0}=\psi_{0}=\operatorname{id}_{B^{2}}$ задают один и тот же элемент группы $\widetilde{\mathscr{G}}$ тогда и только тогда, когда $\varphi_{1}=\psi_{1}$ и пути $\varphi_{t}(1,0)$ и $\psi_{t}(1,0)$ гомотопны на окружности $\partial B^{2}$ относительно концов. Имеем каноническую проекцию $p: \widetilde{\mathscr{G}} \rightarrow \mathscr{G}, \widetilde{\varphi} \mapsto \varphi_{1}$.

ОПРедЕЛЕниЕ 1. Функция $I: G \rightarrow \mathbb{R}$ на группе $G$ называется инвариантом сопряженности, если $I\left(g h g^{-1}\right)=I(h)$ для любых элементов $g, h \in G$.

Пример 1. Инвариант Калаби [3] на группе $\widetilde{\mathscr{G}}$ определяется формулой

$$
\operatorname{Cal}(\widetilde{\varphi}):=\int_{0}^{1}\left(\int_{B^{2}} H_{t} \omega\right) d t, \quad \widetilde{\varphi} \in \tilde{\mathscr{G}}
$$

где $H_{t} \in C_{0}^{\infty}\left(B^{2}, \partial B^{2}\right), 0 \leqslant t \leqslant 1,-$ семейство функций (1), отвечающее кусочно-гладкому семейству симплектоморфизмов $\varphi_{t} \in \mathscr{G}, 0 \leqslant t \leqslant 1, \varphi_{0}=\operatorname{id}_{B^{2}}$, задающему элемент $\widetilde{\varphi} \in \widetilde{\mathscr{G}}$. Нетрудно показать, что функция $\mathrm{Cal}: \widetilde{\mathscr{G}} \rightarrow \mathbb{R}$ определена корректно и является гомоморфизмом в абелеву группу $(\mathbb{R},+)$, а потому является инвариантом сопряженности. Другие известные инварианты сопряженности на $\widetilde{\mathscr{G}}-$ норма Хофера [4], количество или площадь множества неподвижных точек, спектральные инварианты.

Работа выполнена при поддержке Российского фонда фундаментальных исследований (грант № 12-01-00748-а) и Программы поддержки ведущих научных школ РФ (грант № НШ-581.2014.1).

DOI: $10.4213 / \mathrm{mzm} 10459$ 
2. Дифференцируемые функции на группе $\widetilde{\mathscr{G}}$. Мотивировка следующего определения такова. Группа $\widetilde{\mathscr{G}}$ - это "бесконечномерная группа Ли", причем "касательное пространство" $T_{\widetilde{\varphi}} \widetilde{\mathscr{G}}$ в любой его точке $\widetilde{\varphi}$ состоит из всех гамильтоновых (или бездивергентных) векторных полей с функциями Гамильтона $H \in C_{0}^{\infty}\left(B^{2}, \partial B^{2}\right)$. Поэтому векторное пространство $T_{\widetilde{\varphi}} \widetilde{\mathscr{G}}=S \operatorname{Vect}\left(B^{2}\right)$ можно отождествить с пространством $C_{0}^{\infty}\left(B^{2}, \partial B^{2}\right)$.

ОПредЕление 2. Функцию $I: \widetilde{\mathscr{G}} \rightarrow \mathbb{R}$ назовем дифференцируемой в точке $\widetilde{\varphi} \in \widetilde{\mathscr{G}}$, если существует линейный вещественнозначный функционал на векторном пространстве

$$
C_{0}^{\infty}\left(B^{2}, \partial B^{2}\right) \approx S \operatorname{Vect}\left(B^{2}\right)=T_{\widetilde{\varphi}} \widetilde{\mathscr{G}}
$$

обозначаемый через $d I_{\widetilde{\varphi}}$ и называемый производной функции $I$ в точке $\widetilde{\varphi}$, такой, что для любого гладкого семейства симплектоморфизмов $\varphi_{t} \in \mathscr{G},-\varepsilon<t<\varepsilon$, со свойством $\varphi_{0}=$ $\operatorname{id}_{B^{2}}$ и для соответствующего семейства функций $H_{t} \in C_{0}^{\infty}\left(B^{2}, \partial B^{2}\right),-\varepsilon<t<\varepsilon$, выполнено

$$
\left.\frac{d}{d t}\right|_{t=0} I\left(\widetilde{\varphi}_{t} \widetilde{\varphi}\right)=d I_{\widetilde{\varphi}}\left(H_{0}\right) .
$$

Здесь $\varepsilon>0$, через $\widetilde{\varphi}_{t}$ обозначен элемент группы $\widetilde{\mathscr{G}}$, задаваемый семейством симплектоморфизмов $\varphi_{\tau} \in \mathscr{G}, 0 \leqslant \tau \leqslant t$, если $t \geqslant 0$ (семейством $\varphi_{-\tau} \in \mathscr{G}, 0 \leqslant \tau \leqslant-t$, если $t<0$ ). Производную $d I_{\widetilde{\varphi}}$ назовем регулярной, если она является регулярным элементом двойственного пространства $C_{0}^{\infty}\left(B^{2}, \partial B^{2}\right)^{*}$, т.е. ее значение на любой функции $H \in C_{0}^{\infty}\left(B^{2}, \partial B^{2}\right)$ имеет вид

$$
d I_{\widetilde{\varphi}}(H)=\int_{B^{2}} K_{I}(\widetilde{\varphi}, x, y) H(x, y) d x d y
$$

для некоторой измеримой функции $K_{I}(\widetilde{\varphi}, \cdot)$ в круге $B^{2}$, называемой плотностью функционала $d I_{\widetilde{\varphi}}$.

ОПредЕлЕниЕ 3. Пусть функция $I: \widetilde{\mathscr{G}} \rightarrow \mathbb{R}$ дифференцируема всюду на $\widetilde{\mathscr{G}}$ и имеет регулярную производную. Будем говорить, что производная непрерывна относительно $C^{k}$-топологии на $\widetilde{\mathscr{G}}$ (для $\left.k \geqslant 0\right)$, если ее плотность $K_{I}=K_{I}(\widetilde{\varphi}, x, y)$ непрерывна на $\widetilde{\mathscr{G}} \times B^{2}$ относительно топологии прямого произведения, где группа $\widetilde{\mathscr{G}}$ снабжена $C^{k}$-топологией.

Пример 2. Инвариант Калаби дифференцируем всюду на $\widetilde{\mathscr{G}}$. Его производная регулярна и имеет постоянную плотность $K_{\mathrm{Cal}}=1$. Поэтому его производная непрерывна относительно любой топологии на группе $\widetilde{\mathscr{G}}$.

3. Основной результат. Наш результат аналогичен следующим результатам. Любой гомоморфизм группы $\widetilde{\mathscr{G}}$ в группу $(\mathbb{R},+)$ имеет вид $h \circ \mathrm{Cal}$ для некоторого гомоморфизма $h: \mathbb{R} \rightarrow \mathbb{R}[5]$. Любой инвариант "первого порядка" уравнения Эйлера идеальной несжимаемой жидкости в ограниченной области в $\mathbb{R}^{3}$ выражается через энергию и спиральность [6].

Теорема 1. Пусть $I: \widetilde{\mathscr{G}} \rightarrow \mathbb{R}-$ инвариант сопряженности на группе $\widetilde{\mathscr{G}}$, дифферениируемый всюду на $\widetilde{\mathscr{G}}$ и имеющий регулярную и непрерывную производную относительно $C^{1}$-топологии на $\widetilde{\mathscr{G}}$. Тогда этот инвариант выражается через инвариант Калаби, т.е. имеет вид $I=h \circ$ Cal для некоторой функции $h: \mathbb{R} \rightarrow \mathbb{R}$.

ЛЕмма. Пустъ $I: \widetilde{\mathscr{G}} \rightarrow \mathbb{R}-$ инвариант сопряженности, дифферениируемый в точке $\widetilde{\varphi} \in \widetilde{\mathscr{G}}$. Тогда для любого элемента $\widetilde{\psi} \in \widetilde{\mathscr{G}}$, коммутирующего с $\widetilde{\varphi}$, и любой функиии $H \in$ $C_{0}^{\infty}\left(B^{2}, \partial B^{2}\right)$ выполнено равенство $d I_{\widetilde{\varphi}}(H)=d I_{\widetilde{\varphi}}(H \circ \psi)$, где $\psi:=p(\widetilde{\psi}) \in \mathscr{G}, p: \mathscr{G} \rightarrow \mathscr{G}-$ проекиия.

ДокАЗАТЕЛЬСтво. Действительно, в обозначениях определения 2 имеем

$$
I\left(\widetilde{\varphi}_{t} \widetilde{\varphi}\right)=I\left(\widetilde{\psi}^{-1} \widetilde{\varphi}_{t} \widetilde{\varphi} \widetilde{\psi}\right)=I\left(\widetilde{\psi}^{-1} \widetilde{\varphi}_{t} \widetilde{\psi} \widetilde{\varphi}\right)=I\left(\widetilde{\chi}_{t} \widetilde{\varphi}\right)
$$


где $\widetilde{\chi}_{t}:=\widetilde{\psi}^{-1} \widetilde{\varphi}_{t} \widetilde{\psi}$. Семейству $\widetilde{\chi}_{t}$ отвечает семейство функций $H_{t} \circ \psi \in C_{0}^{\infty}\left(B^{2}, \partial B^{2}\right)$. Дифференцируя доказанное соотношение $I\left(\widetilde{\varphi}_{t} \widetilde{\varphi}\right)=I\left(\widetilde{\chi}_{t} \widetilde{\varphi}\right)$ по $t$ при $t=0$, получаем требуемое равенство для $H=H_{0}$. Лемма доказана.

СлеДСтвиЕ. Предположим, что в условиях леммы производная $d I_{\widetilde{\varphi}}$ в точке $\widetilde{\varphi}$ регулярна и ее плотность $K_{I}(\widetilde{\varphi}, \cdot)$ непрерывна на круге $B^{2}$. Тогда верно равенство $K_{I}(\widetilde{\varphi}, \cdot)=$ $K_{I}(\widetilde{\varphi}, \psi(\cdot))$ на круге $B^{2} . \quad B$ частности, $K_{I}(\widetilde{\varphi}, \cdot)=K_{I}(\widetilde{\varphi}, \varphi(\cdot))$, т.е. функция $K_{I}(\widetilde{\varphi}, \cdot)$ постоянна на любой $\varphi$-орбите, а потому и на замыкании любой $\varphi$-орбитъ в круге $B^{2}$.

ДокАзАтЕльство. Действительно, равенство из леммы переписывается в виде

$$
\int_{B^{2}} K_{I}(\widetilde{\varphi}, \cdot) H \omega=\int_{B^{2}} K_{I}(\widetilde{\varphi}, \cdot)(H \circ \psi) \omega, \quad H \in C_{0}^{\infty}\left(B^{2}, \partial B^{2}\right) .
$$

Так как диффеоморфизм $\psi \in \mathscr{G}$ сохряняет 2-форму $\omega$, левая часть этого равенства равна $\int_{B^{2}} K_{I}(\widetilde{\varphi}, \psi(\cdot))(H \circ \psi) \omega$. Так как полученное равенство верно для любой функции $H \in$ $C_{0}^{\infty}\left(B^{2}, \partial B^{2}\right)$ и функция $K_{I}(\widetilde{\varphi}, \cdot)$ непрерывна на круге $B^{2}$, получаем требуемое равенство. Следствие доказано.

ДОКАЗАТЕЛЬСТВо ТЕОРЕМЫ 1. ПредПоложим, что симплектоморфизм $\varphi=p(\widetilde{\varphi}) \in \mathscr{G}$ топологически транзитивен, т.е. его орбита всюду плотна в круге $B^{2}$. Так как замыкание этой орбиты совпадает с кругом $B^{2}$, в силу следствия функция $K_{I}(\widetilde{\varphi}, \cdot)$ постоянна в круге $B^{2}$. Согласно результату [7; теорема 3.1$]$ из консервативной динамики множество топологически транзитивных элементов $C^{1}$-плотно в $\mathscr{G}$ (и, более того, является $C^{1}$-мощным, т.е. содержит пересечение счетного семейства $C^{1}$-плотных $C^{1}$-открытых подмножеств). Значит, для "почти всех" (т.е. образующих $C^{1}$-мощное подмножество в $\widetilde{\mathscr{G}}$ ) элементов $\widetilde{\varphi} \in \tilde{\mathscr{G}}$ функция $K_{I}(\widetilde{\varphi}, \cdot)$ постоянна в круге $B^{2}$.

По условию теоремы непрерывная функция $K_{I}(\widetilde{\varphi}, \cdot)$ на круге $B^{2}$ непрерывно зависит от элемента $\widetilde{\varphi} \in \widetilde{\mathscr{G}}$ относительно $C^{1}$-топологии на $\widetilde{\mathscr{G}}$. Но по доказанному выше эта функция постоянна в круге $B^{2}$ для "почти всех" (в смысле $C^{1}$-топологии) элементов $\widetilde{\varphi} \in \widetilde{\mathscr{G}}$. Поэтому она постоянна в круге $B^{2}$ для всех элементов $\widetilde{\varphi} \in \widetilde{\mathscr{G}}$, т.е. является константой $K_{I}(\widetilde{\varphi}):=$ $K_{I}(\widetilde{\varphi}, x, y)$ на круге, не зависящей от точки $(x, y) \in B^{2}$.

Имеем

$$
d I_{\widetilde{\varphi}}(H)=K_{I}(\widetilde{\varphi}) \int_{B^{2}} H \omega=K_{I}(\widetilde{\varphi}) d \operatorname{Cal}_{\widetilde{\varphi}}(H) .
$$

Значит, функция $I$ локально постоянна на любом множестве уровня $\mathrm{Cal}^{-1}(c)$ инварианта Калаби, $c \in \mathbb{R}$. Хорошо известно, что любое множество уровня $\mathrm{Cal}^{-1}(c)$ в $\widetilde{\mathscr{G}}$ линейно связно (это очевидно следует из того, что семейства функций (1) со средним значением $c$ образуют выпуклое подмножество). Поэтому на множестве $\mathrm{Cal}^{-1}(c)$ функция $I$ постоянна, т.е. равна некоторой константе $h(c)$, зависящей только от $c \in \mathbb{R}$ (для некоторой функции $h: \mathbb{R} \rightarrow \mathbb{R}$ ). Таким образом, $I(\widetilde{\varphi})=h(\operatorname{Cal}(\widetilde{\varphi}))$ для любого $\widetilde{\varphi} \in \widetilde{\mathscr{G}}$. Теорема 1 доказана.

Аналогично доказывается следующая

Теорема 2. Пусть $I: \widetilde{\mathscr{G}} \rightarrow \mathbb{R}$ - инвариант сопряженности на группе $\widetilde{\mathscr{G}}$, дифференцируемый на $C^{1}$-открытом подмножестве $U \subset \widetilde{\mathscr{G}}$ и имеющий регулярную и непрерывную производную относительно $C^{1}$-топологии на $U \subset \widetilde{\mathscr{G}}$. Тогда инвариант $\left.I\right|_{U}$ постоянен на любой линейно-связной компоненте множества уровня $U \cap \mathrm{Cal}^{-1}(c)$ инварианта Калаби в $U, c \in \mathbb{R}$.

Известно [8], [9] описание инвариантов сопряженности на множестве "невырожденных интегрируемых симплектоморфизмов" в группе гомеоморфизмов $\operatorname{Homeo}\left(M^{2}\right) \supset S \operatorname{Diff}\left(M^{2}\right)$, где $M^{2}$ - замкнутая ориентируемая поверхность, снабженная формой площади $\omega$. (Указанное множество состоит из отображений за время 1 потоков гамильтоновых векторных полей на $\left(M^{2}, \omega\right)$ с морсовскими функциями Гамильтона $H \in C^{\infty}\left(M^{2}\right)$, не зависящими от 
времени.) Автором исследовалась [10], [11] непрерывность этих инвариантов (и их комбинаций) относительно $C^{\infty}$-топологии на $C^{\infty}\left(M^{2}\right)$.

Автор выражает благодарность Г. Хорнигу и Х. Цишангу за полезные обсуждения и организацию в 2000-2001 г. семинара по топологическим инвариантам магнитных полей, Л. В. Полтеровичу за указание на работу [7].

\section{СПИСОК ЦИТИРОВАННОЙ ЛИТЕРАТУРЫ}

[1] J. Moser, Trans. Amer. Math. Soc., 120 (1965), 286-294. [2] S. Smale, Proc. Amer. Math. Soc., 10:4 (1959), 621-626. [3] E. Calabi, Problems in Analysis, Princeton Univ. Press, Princeton, NJ, 1970, 1-26. [4] H. Hofer, Comment. Math. Helv., 68:1 (1993), 48-72. [5] A. Banyaga, Comment. Math. Helv., 53:2 (1978), 174-227. [6] D. Serre, C. R. Acad. Sci. Paris Sér. A, 289:4 (1979), 267-270; Phys. D, 13:1-2 (1984), 105-136. [7] C. Bonatti, S. Crovisier, Invent. Math., 158:1 (2004), 33-104. [8] A. V. Bolsinov, A. T. Fomenko, Дифференииалънал геометрия, группь Ли и механика. 15-2, Зап. научн. сем. ПОМИ, 235, ПОМИ, СПб., 1996, 22-53. [9] А. В. Болсинов, А. Т. Фоменко, Изв. РАН. Сер. матем., 59:1 (1995), 65-102. [10] Е. А. Кудрявцева, Докл. РАН, 361:3 (1998), 314-317. [11] Ю. А. Браилов, Е. А. Кудрявцева, Вестн. Моск. ун-та. Сер. 1. Матем., мех., 1999, № 2, 20-27.

\section{Е. А. Кудрявцева}

Московский государственный университет им. М. В. Ломоносова

E-mail: eakudr@mech.math.msu.su
Поступило 09.01.2014 